Classification

Physics Abstracts

61.14-x - 61.16Di

\title{
Convergent Beam Electron Diffraction Analysis of Strain in Multilayer Structures: A Kinematical Approach
}

\author{
Daniela Manno and Saverio Mongelli \\ Dipartimento di Scienza dei Materiali, Università di Lecce, Via Arnesano, 73100 Lecce, Italy
}

(Received March 1; accepted July 28, 1995)

\begin{abstract}
Convergent beam electron diffraction (CBED) performed on plan-view samples has been considered in order to analyse strained superlattices. The composition modulation along the growth axis of multilayer materials and the strain modulation at the interfaces give rise to special features in higher order Laue zone (HOLZ) reflections. A simple model based on the expansioncontraction of the lattice spacing in each layer along the growth direction is proposed to simulate rocking curves in the kinematical approximation. Applications to $\mathrm{Cd}_{x} \mathrm{Zn}_{1-x} \mathrm{Se} / \mathrm{ZnSe}$ superlattices are discussed.
\end{abstract}

\section{Introduction}

In recent years semiconductor superlattices have become very important in semiconductor science and technology. To understand the electrical and optical properties of such materials and to improve growth procedures accurate structural characterization is needed.

So far structural characterization (layer thicknesses, interface atomic roughness and sharpness and any layer strain present) was performed by high resolution electron microscopy applied to cross-sectional specimens. However, to interpret correctly high resolution images it is necessary to consider the structural distortions induced by shear stresses. Such distortions can be large and cannot be ignored in electron microscopy studies. More significantly, the distortion is a function of sample thickness and thin-foil stress relaxation effects originate by the sample thinning [1].

Because strain remains essentially unrelaxed in the plan-view geometry, electron rocking curves observed in convergent beam electron diffraction (CBED) patterns allow for strain and layer thickness evaluation of multilayer samples without complications due to thin-foil stress relaxation effects [2-4]. It is well know that the composition modulation along the growth axis of multilayer structures introduces extra higher-order Laue zone (HOLZ) rings, in addition, the strain modulation at the interfaces causes characteristic diffraction effects, such as shifts in HOLZ lines [5].

In order to interpret the effects due to strain in the experimental electron diffraction patterns, a simulation is needed. In this work we present a simple model based on the assumption that an expansion-contraction of the lattice spacing of each layer along the growth direction occurs. 
The scattered amplitude has been calculated as a function of the deviation vector in the kinematical approximation. Finally, the simulated rocking curves were compared to the experimental ones. Despite the dynamical nature of the electron diffraction, the main features in the CBED reflections can be understood using the simple kinematical theory.

\section{Computing Details}

We refer to a generic specimen $\mathrm{A}_{x} \mathrm{~B}_{1-x} \mathrm{C} / \mathrm{BC}$ multilayer as in Figure 1a. Let $N$ be the number of alternating double layers ("a": layer $\mathrm{A}_{x} \mathrm{~B}_{1-x} \mathrm{C}+$ " $\mathrm{b}$ " layer $\mathrm{BC}$ ), $d_{\mathrm{a}}$ and $d_{\mathrm{b}}$ the respective layers thicknesses, $a_{\mathrm{a}}$ and $a_{\mathrm{b}}$ the lattice spacing.

Assuming $a_{\mathrm{a}}>a_{\mathrm{b}}$, the layer $\mathrm{a}$ is compressed and the layer $\mathrm{b}$ expanded in the interface plane $(x-y)$; whereas, in the growth direction $z$ (normal to the interface) the layer a is expanded and the layer $\mathrm{b}$ is compressed by a factor $\phi=(1+\nu) /(1-\nu)$, where $\nu$ is the Poisson ratio.

The layers match at the interface, so no relative displacement exists in this plane; on the contrary, in the $z$-direction contractive and expansive displacements occur. We assume a linear displacement $R(z)$ for unit cells along the growth direction as shown in Figure 1b:

$$
\begin{array}{ll}
R_{\mathrm{a}}(z)=e_{\mathrm{a}}^{\perp}(z-n d) & n d<z<n d+d_{\mathrm{a}} \\
R_{\mathrm{b}}(z)=e_{\mathrm{b}}^{\perp}(z-(n+1) d) & n d+d_{\mathrm{a}}<z<(n+1) d
\end{array}
$$

where $e_{\mathrm{a}}^{\perp}$ and $e_{\mathrm{b}}^{\perp}$ are the strains of the two layers along the $z$-direction which are related to $e_{\mathrm{a}}^{\|}, e_{\mathrm{b}}^{\|}$ (in-plane strains) by:

$$
\begin{aligned}
& e_{\mathrm{a}}^{\perp}=-\phi e_{\mathrm{a}}^{\|} \\
& e_{\mathrm{b}}^{\perp}=-\phi e_{\mathrm{b}}^{\|}
\end{aligned}
$$

The in-plane strains are related to the lattice mismatch of the layers by

$$
e_{\mathrm{b}}^{\|}-e_{\mathrm{a}}^{\|}=f
$$

Because no relative displacement exists in the interface plane, we have:

$$
k_{\mathrm{a}} d_{\mathrm{a}} e_{\mathrm{a}}^{\|}=k_{\mathrm{b}} d_{\mathrm{b}} e_{\mathrm{b}}^{\|}
$$

where $k_{\mathrm{a}}$ and $k_{\mathrm{b}}$ are the elastic constants of the two layers.

From equations (1) to (4), assuming also a low $x$ value (low content of "A" element) we obtains:

$$
\begin{array}{ll}
R_{\mathrm{a}}(z)=\frac{\phi d_{\mathrm{b}} f}{d}(z-n d) & n d<z<n d+d_{\mathrm{a}} \\
R_{\mathrm{b}}(z)=\frac{-\phi d_{\mathrm{a}} f}{d}(z-(n+1) d) & n d+d_{\mathrm{a}}<z<(n+1) d
\end{array}
$$

In the kinematical theory of electron diffraction, the intensity of a reflection $g$ is given by $\left|\phi_{g}\right|^{2}$, where $\phi_{g}=\int F_{g} \exp [-2 \pi(s \tau+g \cdot R)] \mathrm{d} \tau[6]$. 
In our case:

$$
\begin{aligned}
\phi_{g}= & \sum_{n=0}^{N-1} F_{\mathrm{a}} \int_{n d}^{n d^{\prime}+d_{\mathrm{a}}^{\prime}} \exp \left\{-2 \pi i\left[s \tau+g_{z} e_{\mathrm{a}}^{\perp}\left(\tau \cos \vartheta-n d^{\prime}\right)\right]\right\} \mathrm{d} \tau+ \\
& \sum_{n=0}^{N-1} F_{\mathrm{b}} \int_{n d^{\prime}+d_{\mathrm{a}}^{\prime}}^{(n+1) d^{\prime}} \exp \left\{-2 \pi i\left[s \tau+g_{z} e_{\mathrm{b}}^{\perp}\left(\tau \cos \vartheta-(n+1) d^{\prime}\right)\right]\right\} \mathrm{d} \tau
\end{aligned}
$$

$d^{\prime}=d / \cos \vartheta$ and $d_{\mathrm{a}}^{\prime}=d_{\mathrm{a}}^{\prime} / \cos \vartheta$

$\vartheta$ is the angle between [001] and the chosen zone axis.

The scattering factors $F_{\mathrm{a}}$ and $F_{\mathrm{b}}$ were determined by a linear interpolation between the scattering factors of materials $\mathrm{BC}$ and $\mathrm{AC}$, calculated by the EMS program [7] and they are functions of $x$. Similar linear interpolations have been considered in order to determine the elastic constants and strains.

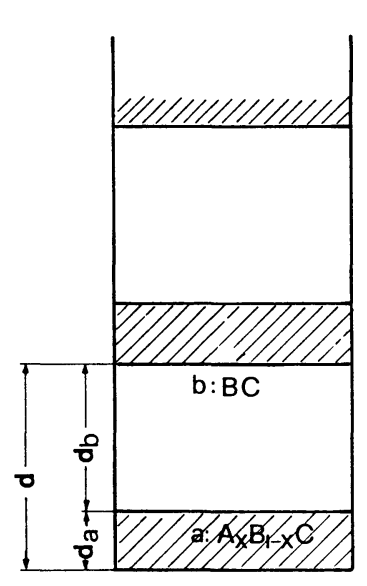

(a)

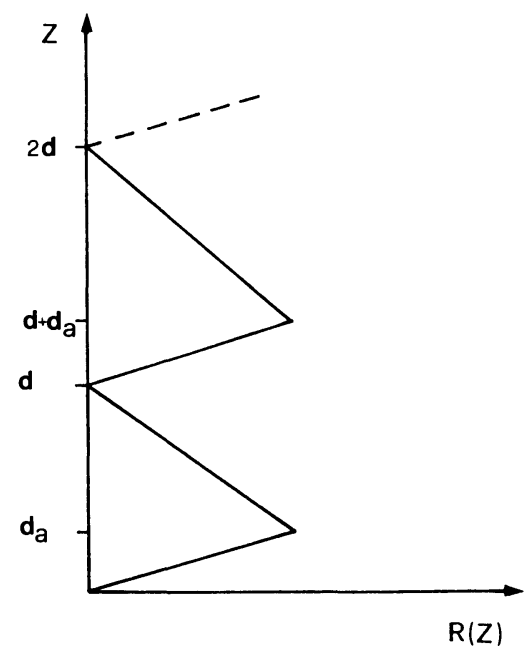

(b)

Fig. 1. - a) Outline of a strained multilayer structure; b) $R(z)$ displacement function describes the contraction and expansion of lattice spacing in the growth direction $z$.

\section{Application and Testing of Model}

The validity of the model has been tested on $\mathrm{Cd}_{x} \mathrm{Zn}_{1-x} \mathrm{Se} / \mathrm{ZnSe}$ multilayers of nominal period $23 \mathrm{~nm}\left(\mathrm{Cd}_{x} \mathrm{Zn}_{1-x}\right.$ Se $\left.3 \mathrm{~nm}, \mathrm{ZnSe} 20 \mathrm{~nm}\right)$, and $x=0.10 \mathrm{Cd}$ content.

CBED investigations were performed in a JEM 2010 transmission electron microscope operating at a nominal $200 \mathrm{kV}$ accelerating voltage. Details on the experiments will be reported elsewhere [8].

Because reflections coming from planes inclined with respect to the interface were asymmetric and showed rocking curves very sensitive both to the strain and to the composition modulation, CBED zone axis diffractions tilted away from [001] growth direction were obtained. 


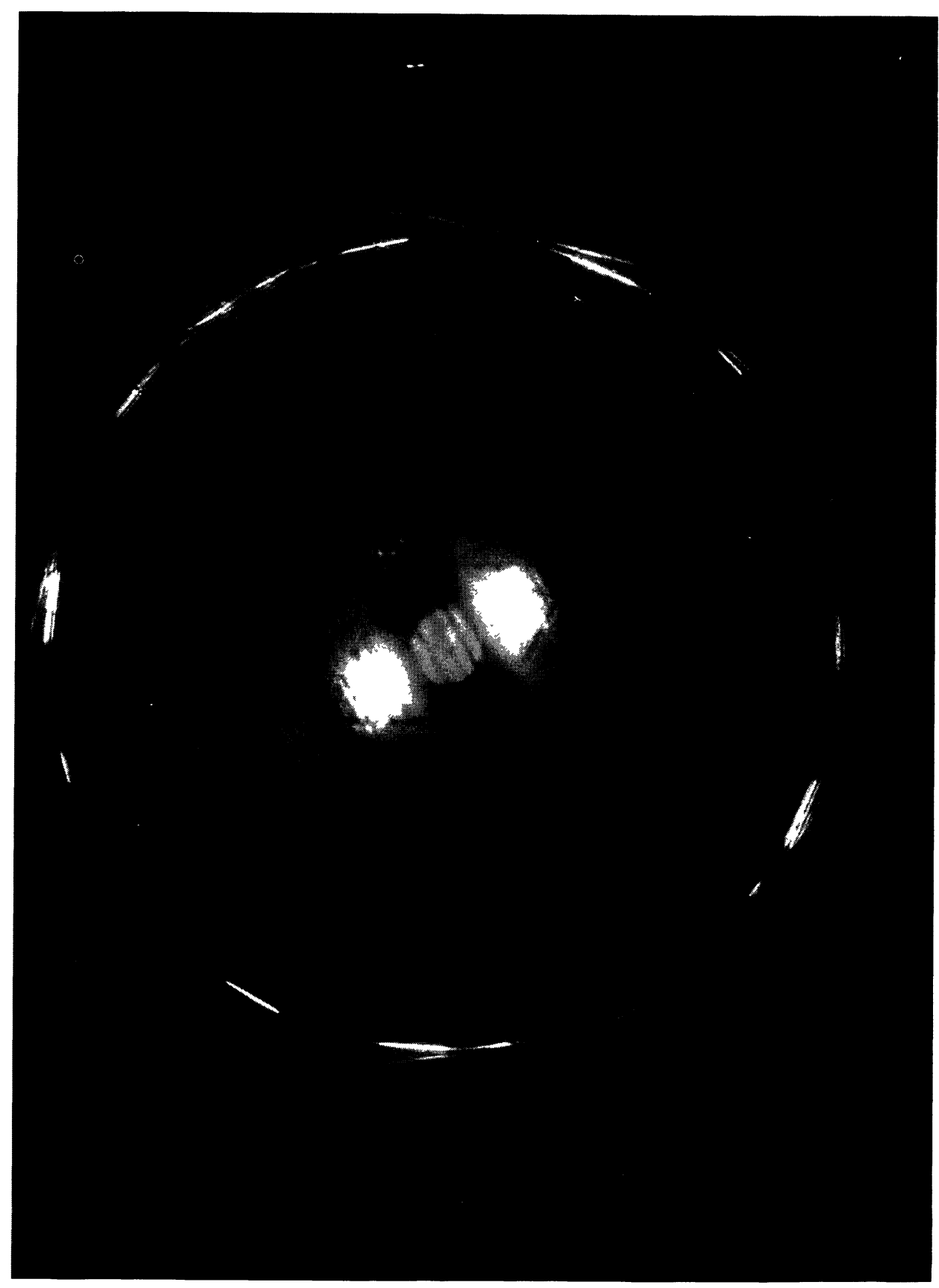

Fig. 2. - [611] CBED zone-axis pattern, the excited HOLZ reflections $\left(\begin{array}{ll}12 & 2 \\ 2\end{array}\right)$ and $(\overline{12} 2 \overline{2})$ are marked.

Figure 2 shows a [611] zone-axis CBED pattern obtained from a $\mathrm{Cd}_{x} \mathrm{Zn}_{1-x} \mathrm{Se} / \mathrm{ZnSe}$ sample in plan view geometry $\left(\mathrm{Cd}_{x} \mathrm{Zn}_{1-x} \mathrm{Se} 3 \mathrm{~nm}, \mathrm{ZnSe} 20 \mathrm{~nm}\right.$ and $x=0.10 \mathrm{Cd}$ content $)$. We concentrate our attention on the reflections (12 22$)$ and $(\overline{12} 2 \overline{2})$; their asymmetry it is evident. In addition, 


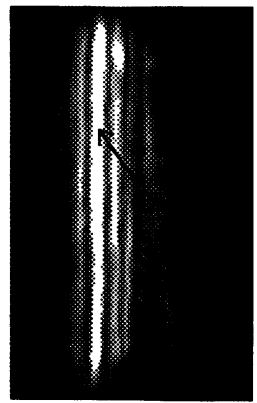

(a)

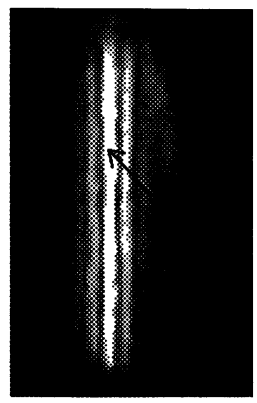

(b)
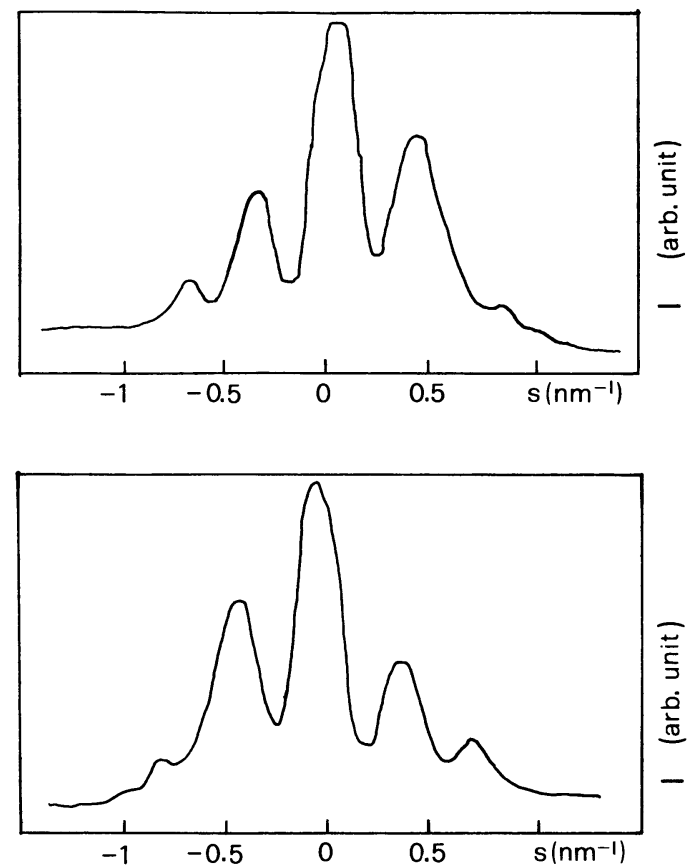

Fig. 3. - Digitized reflections a) $(1222)$ and b) $(\overline{12} 2 \overline{2})$ and corresponding profiles. The arrows on reflections indicate the exact HOLZ ring $(s=0)$ position.

the principal maximum is shifted away from the HOLZ ring. Figure 3 reports digitized images of reflections (12 2 2) (Fig. 3a) and ( $\overline{12} 2 \overline{2}$ ) (Fig. 3b) acquired by a scanner directly on the

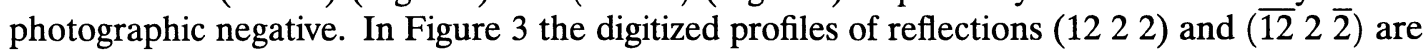
also reported. The plus and minus sign of $s$ refer to sidebands inside and outside the Ewald sphere, corresponding to sidebands outside and inside the HOLZ rings.

The calculated intensity profiles of the HOLZ reflections $\left(\begin{array}{lll}12 & 2 & 2\end{array}\right)$ and $\left(\begin{array}{lll}\overline{12} & 2 & \overline{2}\end{array}\right)$ are shown in Figure 4. It is evident that, as the $\mathrm{Cd} x$ content increase, the calculated profiles change noticeably. In addition, as $x$ increases, the principal maximum shift away from the HOLZ ring, according to experimental data.

The computed and the digitized profiles fit only qualitatively. This occur both because the kinematical approximation has been used to computing the profiles and because the darkness on the photographic negatives (Kodak SO163), used to record the diffraction pattern, is not exactly proportional to the intensity of incoming electrons. Consequently, the comparison of experimental and computed intensity is misleading. However the experimental data and the computed ones fit qualitatively in the range $0.10<x<0.20$. For $x>0.2$, the principal maximum shifts far away from HOLZ ring to match the experimental data. 


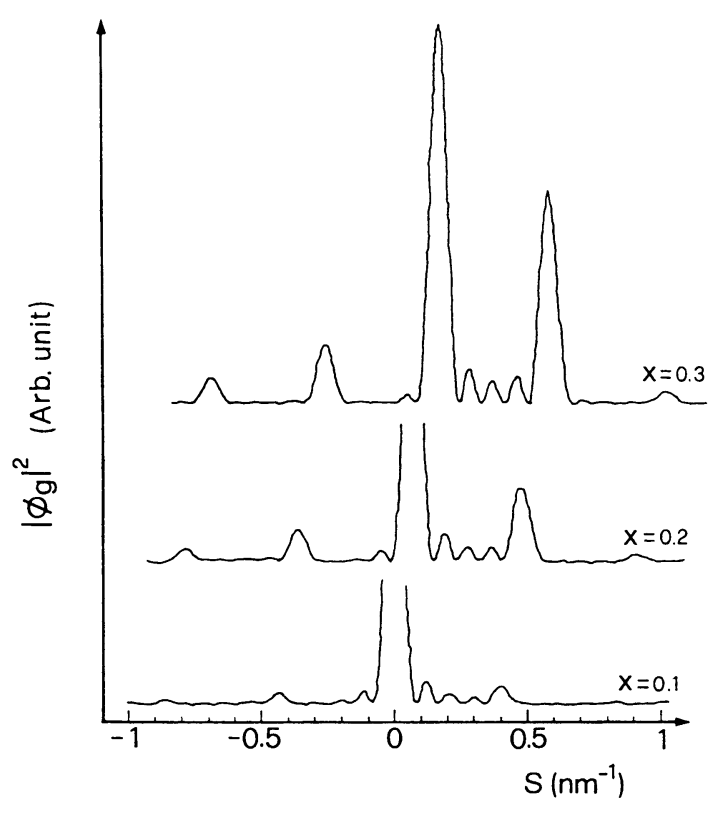

a)

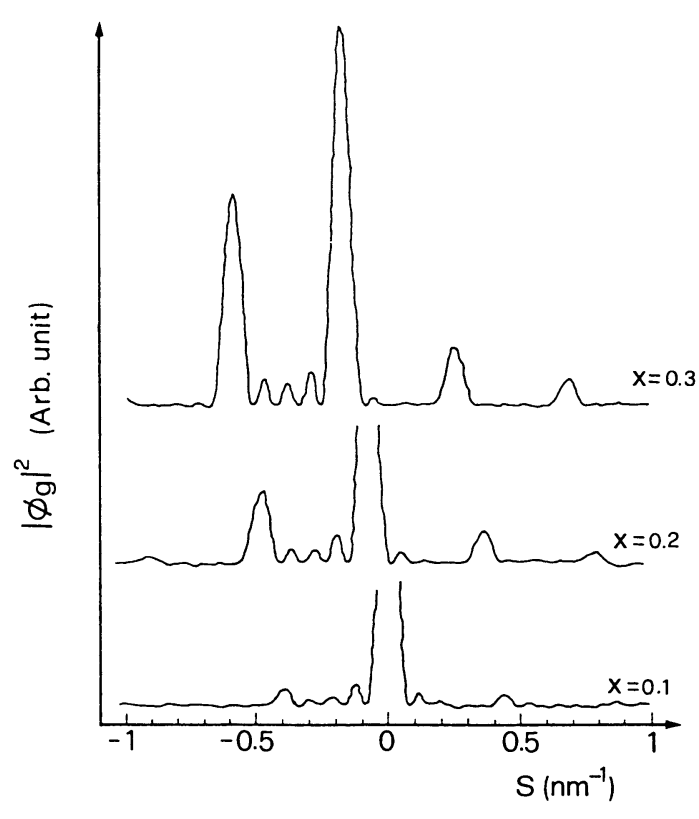

b)

Fig. 4. - Calculated profiles of the HOLZ reflections a) $\left(\begin{array}{lll}12 & 2 & 2\end{array}\right)$ and b) $(\overline{12} 2 \overline{2})$.

\section{Conclusions}

Convergent beam electron diffraction performed on plan-view samples has been considered in order to analyse strained superlattices. The fine details in the HOLZ reflections allow us to analyse conveniently these materials without the complication due to the elastic relaxation effects introduced by the thinning process.

The main features in CBED reflections can be understood using the simple kinematical theory providing a convenient description of the multilayer structure, despite the dynamical nature of electron diffraction. To obtain quantitative results, HOLZ reflections have to be acquired directly in order to have a linear correlation between incoming electrons and digitized intensity.

\section{Acknowledgements}

The author would like to thank Dr. A Franciosi of Laboratori TASC - INFM in Trieste (Italy) for providing the $\mathrm{Cd}_{x} \mathrm{Zn}_{1-x} \mathrm{Se} / \mathrm{ZnSe}$ superlattice, and Prof. R. Cingolani for critical revision of the text. Special thanks to Dr. R. Cataldo for help in computing work. The technical support of Mr. L. Monteduro is also acknowledged. 


\section{References}

[1] Gibson J.M. and Treacy M.M.J., Ultramicrosc. 14 (1984) 345.

[2] Xie, Fung K.K., York P.K., Fernandez G.E., Eades J.A. and Coleman J.J., Appl. Phys. Lett. 57 (1990) 1978.

[3] Cherns, Touaitia, Preston A.R., Rossouw C.J. and Houghton D.C., Philos. Mag. A64 (1991) 397.

[4] Wang S.Q. and Peng L.M., Ultramicrosc. 55 (1994) 68.

[5] Gong H., C.D. and Schapink F.W., Ultramicrosc. 35 (1991) 171.

[6] Hirsch P., Howie A., Nicholson R.P., Pasheley D.W., Whelan M.J., "Electron Microscopy of Thin Crystals" (Robert E. Krieger Publishing Company, Malabar, Florida, 1977).

[7] Stadelmann P., Ultramicrosc. 21 (1987) 131.

[8] Manno D., Cingolani R., Sorba L., Vanzetti L., Franciosi A., "Convergent beam electron diffraction study of $\mathrm{CdZnSe} / \mathrm{ZnSe}$ strained- layer superlattices", 9th International Conference on Microscopy of Semiconducting Materials (20-23 March 1995). 